PHYSICAL REVIEW D 93, 073008 (2016)

\title{
Assessing the role of nuclear effects in the interpretation of the MiniBooNE low-energy anomaly
}

\author{
M. Ericson \\ Université de Lyon, Université Lyon 1, CNRS/IN2P3, IPN Lyon, F-69622 Villeurbanne Cedex, \\ France and Physics Department, Theory Unit, CERN, \\ CH-1211 Geneva, Switzerland \\ M. V. Garzelli \\ II Institute for Theoretical Physics, Hamburg University, Luruper Chaussee 149, \\ D-22761 Hamburg, Germany \\ C. Giunti \\ INFN, Sezione di Torino, Via P. Giuria 1, I-10125 Torino, Italy \\ M. Martini \\ ESNT, CEA-Saclay, IRFU, Service de Physique Nucléaire, \\ F-91191 Gif-sur-Yvette Cedex, France \\ (Received 25 February 2016; published 20 April 2016)
}

\begin{abstract}
We study the impact of the effect of multinucleon interactions in the reconstruction of the neutrino energy on the fit of the MiniBooNE data in terms of neutrino oscillations. We obtain some improvement of the fit of the MiniBooNE low-energy excess in the framework of two-neutrino oscillations and a shift of the allowed region in the $\sin ^{2} 2 \vartheta-\Delta m^{2}$ plane toward smaller values of $\sin ^{2} 2 \vartheta$ and larger values of $\Delta m^{2}$. However, this effect is not enough to solve the problem of the appearance-disappearance tension in the global fit of short-baseline neutrino oscillation data.
\end{abstract}

DOI: 10.1103/PhysRevD.93.073008

\section{INTRODUCTION}

Neutrino masses and mixing are well established by the observations of neutrino oscillations in solar, atmospheric, and long-baseline neutrino oscillation experiments, which are well accommodated in the standard framework of three-neutrino mixing, where the three active neutrinos $\nu_{e}$, $\nu_{\mu}$, and $\nu_{\tau}$ are superpositions of three massive neutrinos $\nu_{1}$, $\nu_{2}$, and $\nu_{3}$ with respective masses $m_{1}, m_{2}$, and $m_{3}$ (see Refs. [1-3]). In this framework, there are two independent squared-mass differences, the small solar $\Delta m_{\mathrm{SOL}}^{2} \simeq 7.5 \times 10^{-5} \mathrm{eV}^{2}$ and the larger atmospheric $\Delta m_{\mathrm{ATM}}^{2} \simeq 2.3 \times 10^{-3} \mathrm{eV}^{2}$, which can be interpreted as $\Delta m_{\mathrm{SOL}}^{2}=\Delta m_{21}^{2}$ and $\Delta m_{\mathrm{ATM}}^{2}=\left|\Delta m_{31}^{2}\right| \simeq\left|\Delta m_{32}^{2}\right|$, with $\Delta m_{k j}^{2}=m_{k}^{2}-m_{j}^{2}$.

However, the reactor [4-6], Gallium [7-11], and LSND [12] anomalies indicate that the neutrino mixing framework may need an extension in order to accommodate

Published by the American Physical Society under the terms of the Creative Commons Attribution 3.0 License. Further distribution of this work must maintain attribution to the author(s) and the published article's title, journal citation, and DOI. short-baseline oscillations ${ }^{1}$ due to at least one additional squared-mass difference, $\Delta m_{\mathrm{SBL}}^{2} \sim 1 \mathrm{eV}^{2}$, which is much larger than $\Delta m_{\mathrm{ATM}}^{2}$ (see the reviews in Refs. [2,13-18]). The reactor antineutrino anomaly [4] is a deficit of the rate of $\bar{\nu}_{e}$-induced events observed in short-baseline reactor neutrino experiments in comparison with that expected from the calculation of the reactor neutrino fluxes $[5,6]$. The Gallium neutrino anomaly [7-11] is a shortage of $\nu_{e}$-induced events measured at an average distance of about $1 \mathrm{~m}$ in the Gallium radioactive source experiments GALLEX [19] and SAGE [20] with respect to the rate of $\nu_{e}$-induced events expected from the well-measured activity of the radioactive source. The LSND anomaly is the observation of short-baseline $\bar{\nu}_{\mu} \rightarrow \bar{\nu}_{e}$ transitions [12,21].

\footnotetext{
${ }^{1}$ Short-baseline neutrino oscillation experiments are characterized by a ratio $L / E_{\nu} \lesssim 10 \mathrm{~m} \mathrm{MeV}^{-1}$, where $L$ is the sourcedetector distance and $\widetilde{E}_{\nu}$ is the neutrino energy. Since the oscillations generated by a squared-mass difference $\Delta m^{2}$ are observable for $\Delta m^{2} L / 4 E_{\nu} \gtrsim 1$, short-baseline neutrino oscillation experiments are sensitive to $\Delta m^{2} \gtrsim 10^{-1} \mathrm{eV}^{2}$. On the other hand, long-baseline neutrino oscillation experiments are characterized by a ratio $L / E_{\nu} \gtrsim 100 \mathrm{~m} \mathrm{MeV}^{-1}$ which makes them sensitive to $\Delta m^{2} \lesssim 10^{-2} \mathrm{eV}^{2}$.
} 
The additional squared-mass difference required to explain these anomalies with neutrino oscillations necessitates the existence of at least an additional massive neutrino at the $\mathrm{eV}$ scale. Since from the LEP measurement of the invisible width of the $Z$ boson [22] we know that there are only three active neutrinos, in the flavor basis, the additional massive neutrinos correspond to sterile neutrinos [23], which do not have standard weak interactions.

Sterile neutrinos are singlets of the Standard Model gauge symmetries which can couple to the active neutrinos through the Lagrangian mass term. In practice, there are bounds on the active-sterile mixing, but there is no bound on the number of sterile neutrinos and on their mass scales. Therefore, the existence of sterile neutrinos is investigated at different mass scales. ${ }^{2}$ In this paper, we consider the simplest $3+1$ extension of three-neutrino mixing in which the three standard massive neutrinos $\nu_{1}, \nu_{2}$, and $\nu_{3}$ are assumed to have masses much smaller than $1 \mathrm{eV}$ and there is an additional neutrino $\nu_{4}$ with mass $m_{4} \sim 1 \mathrm{eV}$. In this framework, the squared-mass difference $\Delta m_{\mathrm{SBL}}^{2}=\Delta m_{41}^{2} \sim$ $1 \mathrm{eV}^{2}$ can generate short-baseline oscillations which explain the above-mentioned anomalies (see Ref. [18]). In the flavor basis, besides the three active neutrinos $\nu_{e}, \nu_{\mu}$, and $\nu_{\tau}$, there is a sterile neutrino $\nu_{s}$ which has a large mixing with $\nu_{4}$ and small mixings with $\nu_{1}, \nu_{2}$, and $\nu_{3}$. This implies that the elements of the $4 \times 4$ unitary mixing matrix $U$ must be such that $\left|U_{s k}\right| \ll 1$ for $k=1,2,3$ and $\left|U_{\alpha 4}\right| \ll 1$ for $\alpha=e, \mu, \tau$.

In this paper, we consider the results of the MiniBooNE experiment $[45,46]$, which has been done to check the indication in favor of short-baseline neutrino oscillations given by the LSND anomaly. ${ }^{3}$ The MiniBooNE experiment operated first in the neutrino mode, searching for $\nu_{\mu} \rightarrow \nu_{e}$ transitions. The results "showed no evidence of an excess of electron-like events for neutrino energies above $475 \mathrm{MeV}$ " [45], which cover the same $L / E_{\nu}$ range of the LSND experiment. On the other hand, the data showed "unexplained electron-like events in the reconstructed

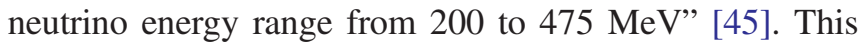
is a sizable excess of $\nu_{e}$-like events in the three energy bins below $475 \mathrm{MeV}$ which has been called the MiniBooNE low-energy anomaly.

\footnotetext{
${ }^{2}$ For example, very light sterile neutrinos at a mass scale smaller than $0.1 \mathrm{eV}$, which could affect the oscillations of solar [24-26] and reactor [27-34] neutrinos; sterile neutrinos at the $\mathrm{keV}$ scale, which could constitute warm dark matter according to the neutrino minimal Standard Model ( $\nu$ MSM) [35] (see the reviews in Refs. [36,37]); sterile neutrinos at the MeV scale [38-41]; and sterile neutrinos at the electroweak scale [42,43] or above it $[43,44]$, the effects of which may be seen at LHC and other high-energy colliders.

${ }^{3}$ The reactor and Gallium anomalies will be checked in a few years by several reactor and radioactive source experiments (see Refs. [18,47])
}

The second part of the MiniBooNE experiment was operated in the antineutrino mode, searching for $\bar{\nu}_{\mu} \rightarrow \bar{\nu}_{e}$ transitions. The final results [46] showed a small excess of $\bar{\nu}_{e}$-like events over the background for reconstructed neutrino energies above $475 \mathrm{MeV}$ and a sizable excess of $\bar{\nu}_{e}$-like events in the three energy bins below $475 \mathrm{MeV}$ which is compatible with the low-energy anomaly in the neutrino mode.

The authors of Refs. $[48,49]$ suggested that at least part of the MiniBooNE low-energy excess could be due to events which have a larger neutrino energy and are interpreted as low-energy events because the reconstruction of the neutrino energy from the measured electron energy and scattering angle did not take into account multinucleon interactions in the neutrino-nucleus chargedcurrent interactions.

The multinucleon emission channel has attracted much attention in the last few years. The inclusion of this channel in the quasielastic cross section was suggested $[50,51]$ as the possible explanation of the MiniBooNE charged-current quasielastic total cross section on carbon [52], observed to be too large with respect to theoretical predictions employing the standard value of the axial mass. The MiniBooNE experiment, as well as other experiments involving Cherenkov detectors, defines as a chargedcurrent quasielasticlike event one in which only a final charged lepton is detected. The ejection of a single nucleon (a genuine quasielastic event) is only one possibility, and one must consider as well events involving, for instance, a correlated nucleon pair from which the partner nucleon is also ejected, as discussed first in Refs. [53]. The inclusion in the quasielastic cross section of events in which several nucleons are ejected ( $n p-n h$ excitations) leads to an increase over the genuine quasielastic value. The authors of Refs. [50,51] argued that this is the likely explanation of the MiniBooNE data, showing that their evaluation can account for the excess in the cross section without any modification of the axial mass. This suggestion triggered a new interest of the neutrino scattering and oscillation communities for the multinucleon emission channel. Beyond the first MiniBooNE data [52], the appearance of new measurements of charged-current quasielasticlike cross sections [54-58], of analyses of the hadronic final states [59-61], and of the vertex and recoil energies deposited in the detector $[55,56,62]$ is leading to mounting experimental evidence of the multinucleon effects in neutrino-nucleus scattering. Several theoretical works [50,51,63-77] have analyzed the role of the multinucleon excitations in the evaluation of the neutrino-nucleus cross sections at MiniBooNE, T2K, and MINERvA energies. Originally, this channel was not included in the Monte Carlo generators used for the analyses of the neutrino cross sections and oscillations experiments. Today, there is an effort to include this $n p-n h$ channel in several event generators [78-81]. As was discussed in 
Refs. [48,49,82,83], the influence of the multinucleon channel also manifests in the problem of the neutrino energy reconstruction [48,49,82-89]. The authors of Refs. [48,49] also showed how it affects the analysis of neutrino oscillation experiments. Applied to the MiniBooNE data, this is the object of the present work.

In this paper, we study the impact of the multinucleon interactions in neutrino-nucleus charged-current scattering on the fit of the electron appearance MiniBooNE data in terms of neutrino oscillations. The MiniBooNE Collaboration discussed briefly an approximate implementation of the multinucleon interactions in Ref. [46]. They showed that the change of the minimum $\chi^{2}$ value is small, and they concluded that multinucleon interactions do not significantly change the oscillation fit. However, the change of the minimum $\chi^{2}$ value due to multinucleon interactions obtained by the MiniBooNE Collaboration is a small increase, whereas we expect a decrease from a better fit of the low-energy excess. In this paper, we examine this problem, and we study quantitatively to which extent the results of the oscillation fit of the MiniBooNE data is affected by the multinucleon interactions.

The plan of the paper is as follows. In Sec. II, we describe the method that we adopted in order to take into account the multinucleon contribution to the neutrinonucleus charged-current interactions in the analysis of MiniBooNE data. In Sec. III, we present the results of the fit of MiniBooNE data, taking into account multinucleon interactions in the simplest framework of twoneutrino mixing. In Sec. IV, we discuss the implications of the multinucleon interactions in the analysis of MiniBooNE data for the global fit of short-baseline neutrino oscillation data in the framework of $3+1$ neutrino mixing. Finally, in Sec. V, we present our conclusions.

\section{METHOD}

In principle, the multinucleon interactions should be included in the Monte Carlo generator with which one simulates the events predicted in the experiment without and with neutrino oscillations. However, since we do not have access to the MiniBooNE Monte Carlo generator, we adopted an approach which allows the treatment of the multinucleon emission channel as well as the quasielastic and the pion production channels through the theoretical model of Ref. [50]. This model has been successful $[50,51,66,71]$ to reproduce the MiniBooNE data on the neutrino [52] and antineutrino [54] quasielasticlike cross sections as well as the pion production data measured by MiniBooNE [90] and the T2K data on muon-neutrino [91] and electron-neutrino [92] inclusive cross sections, as shown in Refs. $[73,77]$. This model is based on the nuclear response functions. The quasielastic response is treated in the random phase approximation, as illustrated, for example, in Ref. [93]. The multinucleon contribution is deduced from the microscopic evaluation of Alberico et al. [94] of the role of two particle-two hole (2p-2h) excitations in the inclusive $\left(e, e^{\prime}\right)$ transverse response. This calculation includes the correlation term; the two-body meson exchange currents terms, in particular the one associated with Delta excitation; and the interference between these quantities. The single-pion production is assumed to arise exclusively from the pionic decay of the Delta excitation. In the nucleus, the Delta width is modified by medium effects. They were introduced and discussed by Oset and Salcedo in Ref. [95]. The nonpionic decay of the Delta in the medium, which modifies its width, leads to $2 \mathrm{p}-2 \mathrm{~h}$ or $3 \mathrm{p}-3 \mathrm{~h}$ excitations contributing to the multinucleon excitations. The parametrization of Ref. [95] for the Delta width in the nuclear medium is used in the model of Ref. [50].

We considered the muon-to-electron neutrino and antineutrino Monte Carlo full transmutation events in the MiniBooNE data release for the final results of the experiment [96]. The corresponding correlation between the true neutrino energy $E_{\nu}$ and the reconstructed one $E_{\nu}^{\text {rec }}$ is shown in the two upper scatter plots in Fig. 1. One can see that most of the points are near the diagonal, which corresponds to the quasielastic energy reconstruction

$$
E_{\nu}^{\mathrm{QE}}=\frac{2\left(M-E_{\mathrm{B}}\right) E_{e}-\left(m_{e}^{2}-2 M E_{\mathrm{B}}+E_{\mathrm{B}}^{2}+\Delta M^{2}\right)}{2\left(M-E_{\mathrm{B}}-E_{e}+p_{e} \cos \theta_{e}\right)},
$$

where $M$ is the mass of the target nucleon which is assumed to be at rest; $E_{\mathrm{B}} \simeq 25 \mathrm{MeV}$ is its binding energy in the nucleus; $E_{e}, p_{e}$, and $\theta_{e}$ are the measured energy, momentum, and scattering angle of the outgoing electron; and $\Delta M^{2}$ is the difference between the squared masses of the initial and final nucleons $\left(\Delta M^{2}=M_{n}^{2}-M_{p}^{2}\right.$ for $\nu_{e}+n \rightarrow$ $p+e^{-}$scattering and $\Delta M^{2}=M_{p}^{2}-M_{n}^{2}$ for $\bar{\nu}_{e}+p \rightarrow n+$ $e^{+}$scattering). The smearing of the quasielastic events around the diagonal in the two upper scatter plots in Fig. 1 is due to the Fermi motion of the initial nucleon and to the electron energy resolution of the detector. In addition, one can note an excess of events with reconstructed energy which is significantly smaller than that in the quasielastic region. These events are due to charged-current pion production $\quad\left(\nu_{e}+n \rightarrow \Delta^{+}+e^{-} \rightarrow n+\pi^{+}+e^{-} \quad\right.$ and $\left.\bar{\nu}_{e}+p \rightarrow \Delta^{0}+e^{+} \rightarrow p+\pi^{-}+e^{+}\right)$. A fraction of the produced pions is not visible because the pions are absorbed in the nucleus on their way out. This process is denoted as the final-state interaction effect [97,98]. Although its presence has not been displayed [97,98] in the neutrino-induced charged pion production MiniBooNE data [90], this process is present in the cross sections of physical pions [50] and is expected to be relevant also in the neutrino reactions. For those pions which do not come out, no tracks other than the lepton ones are visible, and the process is misidentified as a quasielastic event. In their works on the reconstitution problem [48,49], the authors did not consider the unidentified pions channel but only the quasielastic and the multinucleon channels. To exploit their 

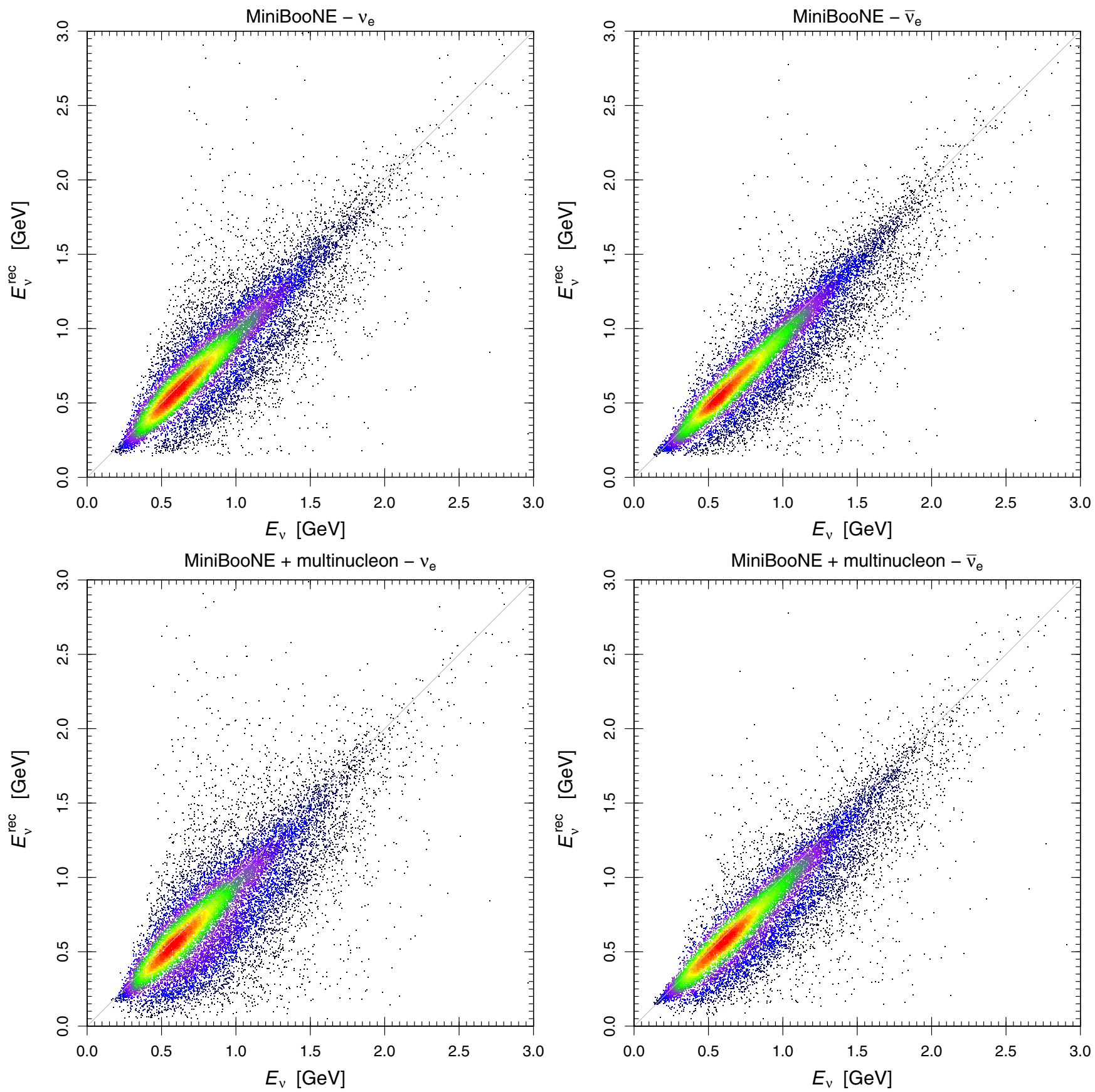

FIG. 1. Scatter plots which show the correlation between the true neutrino energy $E_{\nu}$ and the reconstructed neutrino energy $E_{\nu}^{\text {rec }}$ in the original [96] MiniBooNE muon-to-electron neutrino and antineutrino Monte Carlo full transmutation events (upper plots) and in the events modified with the contribution of multinucleon interactions (lower plots). The color sequence (black, blue, magenta, green, yellow, and red) indicates an increasing density of points.

results for the introduction of the multinucleon channel in the MiniBooNE analysis, we have adopted the following method:

(i) First, we separate the quasielastic events from the pion production events. For this, in the MiniBooNE full transmutation events, we selected statistically the pion production events which are misidentified as quasielastic charged-current events by choosing the events which have a $E_{\nu}-E_{\nu}^{\text {rec }}$ value, which is more likely to be that of a pion production event than that of a quasielastic event. The relative probability of true quasielastic events and misidentified pion production events is calculated with the nuclear model of Ref. [50]. For the estimation of the relative 

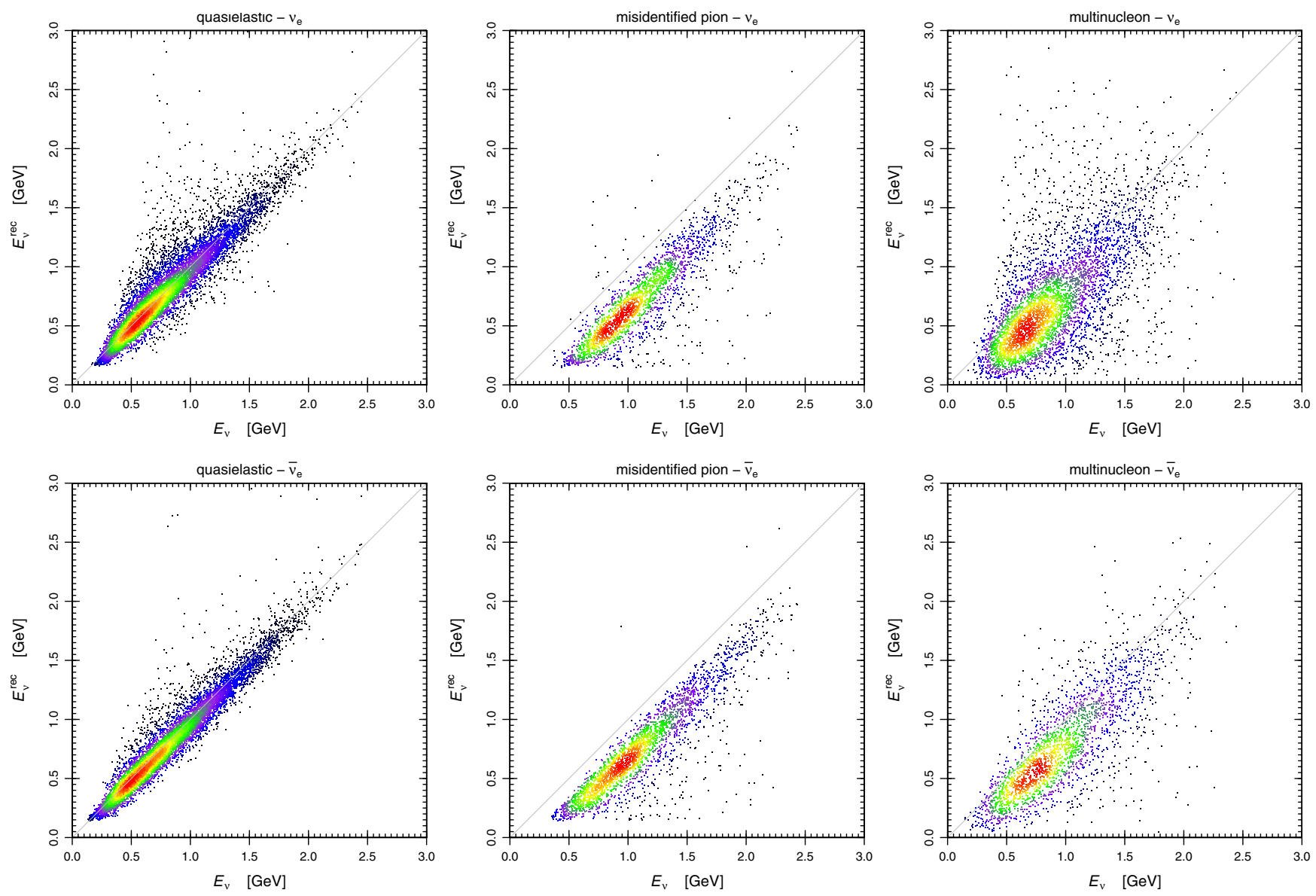

FIG. 2. Scatter plots which shows the correlation between the true neutrino energy $E_{\nu}$ and the reconstructed neutrino energy $E_{\nu}^{\text {rec }}$ separately for quasielastic, misidentified pion, and multinucleon events for neutrino (upper plots) and antineutrino (lower plots) scattering. The color sequence (black, blue, magenta, green, yellow, and red) indicates an increasing density of points.

probability of misidentified pion production events, we considered ${ }^{4} 30 \%$ of the total charged-current single charged pion production events as misidentified quasielastic charged-current events. We do not apply any change to the selected misidentified pion production events.

(ii) We divide randomly the remaining events into a group which we consider as true quasielastic events and a group of events which we transform into multinucleon interaction events. The division is done in proportion to the probability of quasielastic and multinucleon interactions calculated in Refs. [48,49] for different $\left(E_{\nu}, E_{\nu}^{\mathrm{rec}}\right)$ pairs, taking into account that the MiniBooNE detector is filled with pure $\mathrm{CH}_{2}$ mineral oil. Hence, in the neutrino mode $\left(\nu_{e}+n \rightarrow p+e^{-}\right)$, all the scatterings can have a multinucleon contribution because they occur on the neutrons in the carbon atoms, whereas in the

\footnotetext{
${ }^{4}$ We verified that the results do not change in a significant way if we consider a fraction between $20 \%$ and $40 \%$. These percentages are in reasonable agreement with the indications provided in Ref. [97].
}

antineutrino mode $\left(\bar{\nu}_{e}+p \rightarrow n+e^{+}\right)$, only $3 / 4$ of the scatterings are with the protons of the carbon atoms. We do not apply any change to the events in the true quasielastic group.

(iii) We consider the group of multinucleon interaction events for which we calculate a new neutrino reconstructed energy using the theoretical correlation between the true neutrino energy $E_{\nu}$ and the reconstructed neutrino energy $E_{\nu}^{\text {rec }}$ calculated in Refs. [48,49]. We also take into account the energy resolution of the detector given in Fig. 9.19 of Ref. [99].

The results of these transformations are new sets of neutrino and antineutrino Monte Carlo full transmutation events with the correlations between the true neutrino energy $E_{\nu}$ and the reconstructed neutrino energy $E_{\nu}^{\text {rec }}$ shown in the two lower scatter plots in Fig. 1. Comparing them with the corresponding upper scatter plots, one can see that there are more points with reconstructed energy, which is significantly smaller than that in the quasielastic region.

Figure 2 shows the correlation between $E_{\nu}$ and $E_{\nu}^{\text {rec }}$ separately for quasielastic, misidentified pion, and multinucleon events, for neutrino as well as for antineutrino 
scattering. One can notice that the misidentified pion events distribution is displaced horizontally by an amount $\Delta E_{\nu} \simeq$ $300 \mathrm{MeV}$ with respect to the quasielastic one, with a smaller $E_{\nu}^{\text {rec }} / E_{\nu}$ ratio. This shift can be understood by the following argument. The condition for a quasielastic event on a nucleon at rest is $\omega=E_{\nu}-E_{e}=Q^{2} / 2 M_{N}$, where $Q^{2}=|\vec{q}|^{2}-\omega^{2}$ is the squared 4-momentum and $M_{N}$ is the nucleon mass. For Delta excitation on a nucleon, which is responsible for pion production, the condition is, neglecting the Delta width, $\omega=E_{\nu}-E_{e}=Q^{2} / 2 M_{N}+\Delta M$, where $\Delta M=\left(M_{\Delta}^{2}-M_{N}^{2}\right) /$ $\left(2 M_{N}\right) \simeq 338 \mathrm{MeV}$. This is the amount of the horizontal shift of the misidentified pion production distribution. As for the distribution of multinucleon events, it lies between the distribution of quasielastic events peaked on the diagonal and the distribution of misidentified pion events which have the smallest $E_{\nu}^{\text {rec }} / E_{\nu}$ ratio. The multinucleon interactions have the effect of shifting some of the events predicted by neutrino oscillations toward the low-energy bins where the anomalous excess is measured. The effect is larger in neutrino mode because in antineutrino mode the multinucleon interactions are smaller for two reasons. First is the factor 3/4 mentioned before coming from the nature of the target. Moreover, as was explained in Refs. [51,75], in the description of Refs. [50,51], the multinucleon part concerns exclusively the spin-isospin interaction of the weak current with the nucleus. The corresponding spinisospin contribution is reduced for antineutrinos due to the negative value, in this case, of the vector axial interference term.

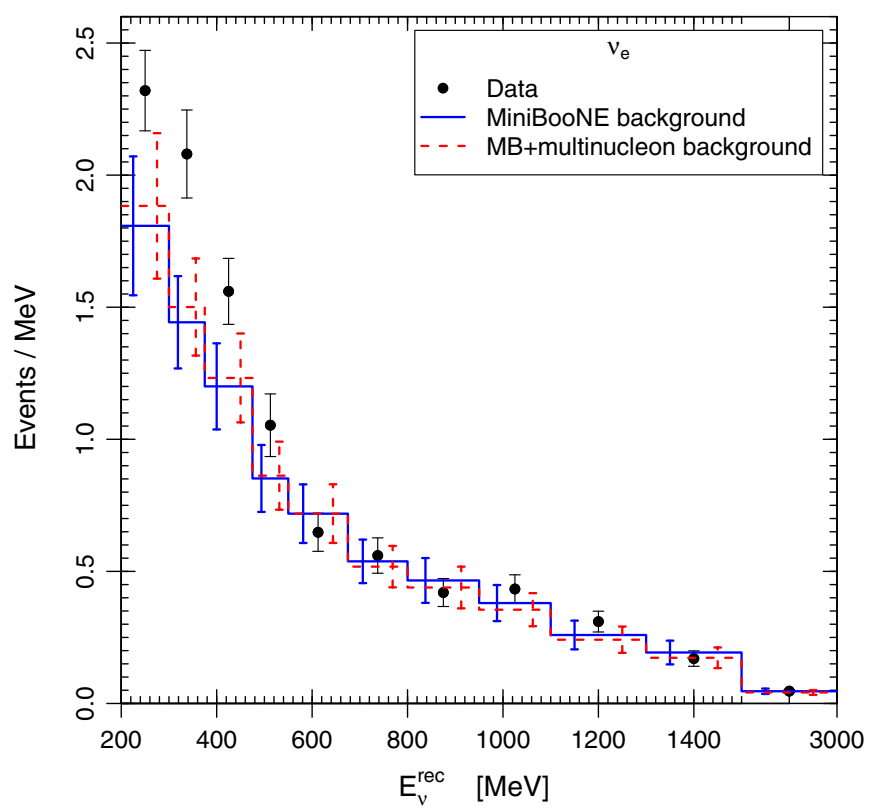

In the analysis of MiniBooNE data, we must also take into account the evaluation of the background, which is

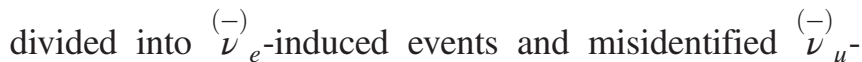
induced events. Since the $\stackrel{(-)}{\nu}_{e}$-induced events are produced by the $\stackrel{(-)}{\nu}_{e}$ 's in the beam generated at the source by pion and kaon decays, their interaction is also affected by the multinucleon contribution. To take it into account, we use the same muon-to-electron neutrino and antineutrino Monte Carlo full transmutation events considered above, taking into account that they are obtained with the $\stackrel{(-)}{\nu}_{\mu}$ flux and the $\stackrel{(-)}{\nu}$ detection cross section. To transform them into events which correspond to a $\stackrel{(-)}{\nu}_{e}$ flux and a $\stackrel{(-)}{\nu}_{e}$ detection cross section, we rescale the weights of each event according to the ratio of $\stackrel{(-)}{\nu}_{e}$ and $\stackrel{(-)}{\nu}_{\mu}$ fluxes in the MiniBooNE beam [100]. We also normalize the weights of the events in each reconstructed energy bin in order to reproduce the number of background $\stackrel{(-)}{\nu}_{e}$-induced events predicted in the MiniBooNE publication [46]. Then, we apply the same method described above in order to add the effect of multinucleon interactions in the calculated back-

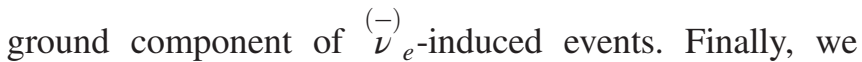
calculate the number of background $\stackrel{(-)}{\nu} e_{e}$-induced events, taking into account the multinucleon interactions by summing the weights of the events in each reconstructed energy bin.

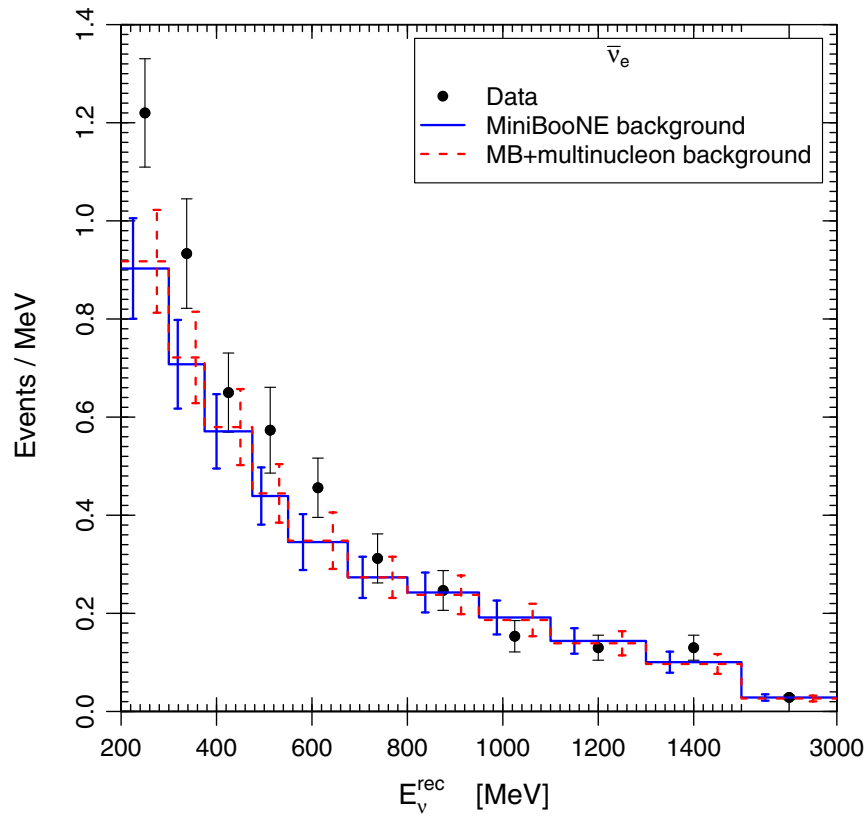

FIG. 3. Comparison between the background in the MiniBooNE data release (solid blue) and that obtained taking into account the multinucleon interactions (dashed red), with the respective systematic uncertainties. The left and right figures correspond, respectively, to the neutrino and antineutrino modes. The data are shown with statistical error bars. 
Figure 3 shows the background histogram as a function of $E_{\nu}^{\text {rec }}$ that we have obtained taking into account multinucleon interactions in comparison with the original background considered by the MiniBooNE Collaboration. One can see that in the neutrino mode the inclusion of the multinucleon interactions leads to a significant increase of the background in the low-energy bins and a small decrease of the background in the high-energy bins. This effect helps to decrease the low-energy anomaly in neutrino mode, as can be seen from the pulls in Table I of the three energy bins below $475 \mathrm{MeV}$ corresponding to the case of no oscillations $\left(\sin ^{2} 2 \vartheta=\Delta m^{2}=0\right)$. In the antineutrino mode, the effect is smaller, and there is little change of the pulls in Table I of the three energy bins below $475 \mathrm{MeV}$ corresponding to the case of no oscillations.

The neutrino oscillation analysis of MiniBooNE is done by using the covariance matrix of systematic uncertainties given in the MiniBooNE data release [96]. This matrix connects the systematic uncertainties of $\stackrel{(-)}{\nu}_{e}$ events in 11 energy bins to those of $\stackrel{(-)}{\nu}_{\mu}$ events in 8 energy bins. The correlation is important because the $\stackrel{(-)}{\nu}_{\mu}$ events, which are measured with high statistics, provide a normalization for the $\stackrel{(-)}{\nu}$ event predictions $[101,102]$. In principle, one should add the multinucleon interactions also to the analysis of the $\stackrel{(-)}{\nu}_{\mu}$ data. However, we could not do it because there is no publicly available sample of Monte Carlo $\stackrel{(-)}{\nu}_{\mu}$ events corresponding to the final MiniBooNE results in the neutrino [45] and antineutrino [46] modes. Moreover, the MiniBooNE Monte Carlo has been tuned in order to fit the high-statistics $\stackrel{(-)}{\nu}_{\mu}$ events $[101,102]$ through the choices of an overall normalization factor 1.28 , the value of the axial mass $m_{A}=1.23 \mathrm{GeV}$, and a nuclear Pauli-blocking factor $\kappa=1.022$. Therefore,

TABLE II. Results of fit of MiniBooNE data without (MiniBooNE) and with (MB + multinucleon) the multinucleon interactions, without neutrino oscillations (No Osc.) and with neutrino oscillations (Osc.) in the simplest framework of two-neutrino mixing. NDF is the number of degrees of freedom and GoF is the goodness of fit.

\begin{tabular}{llcc}
\hline \hline & & MiniBooNE & MB + multinucleon \\
\hline No Osc. & $\chi^{2}$ & 56.0 & 54.7 \\
& NDF & 38 & 38 \\
& GoF & $3 \%$ & $4 \%$ \\
Osc. & $\chi_{\text {min }}^{2}$ & 37.3 & 36.6 \\
& NDF & 36 & 36 \\
& GoF & $41 \%$ & $44 \%$ \\
& $\sin ^{2} 2 \vartheta$ & 1.0 & 0.98 \\
& $\Delta m^{2}$ & 0.040 & 0.041 \\
\hline \hline
\end{tabular}



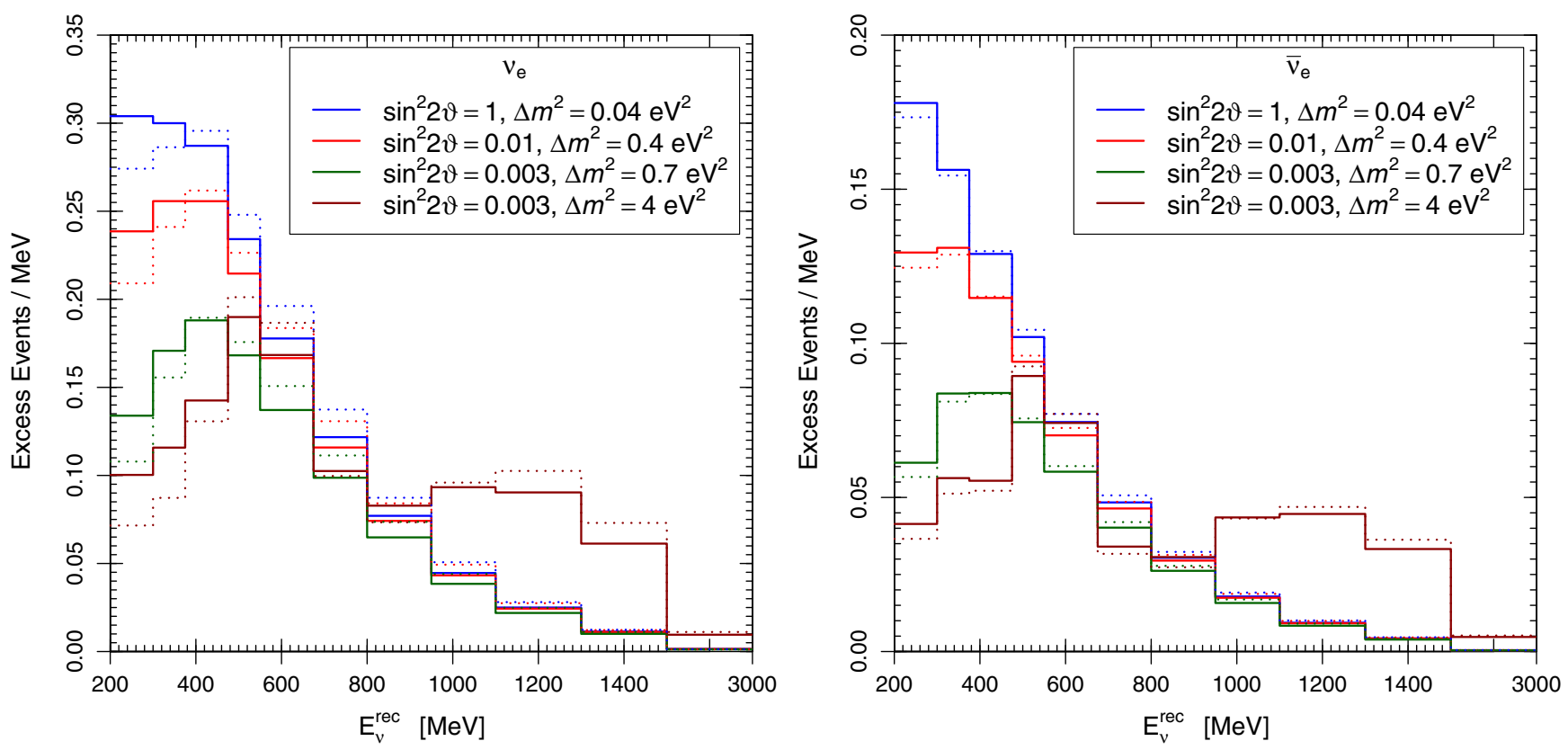

FIG. 4. Comparison of the theoretical excess over the background of $\nu_{e}$ and $\bar{\nu}_{e}$ events in MiniBooNE obtained with neutrino oscillations for some selected values of the oscillation parameters $\sin ^{2} 2 \vartheta$ and $\Delta m^{2}$ without (dotted lines) and with (solid lines) the multinucleon interactions.

our best approach is to neglect possible corrections to the analysis of $\stackrel{(-)}{\nu}_{\mu}$ events due to multinucleon interactions.

The tuning of the MiniBooNE Monte Carlo through a modification of the nucleon axial mass parameter in order to fit the measured $\nu_{\mu}$ cross sections simulating the multinucleon influence naturally leads to the following question: is the multinucleon effect already incorporated in the Monte Carlo $\nu_{e}$ full transmutation events? In this respect, we can make the following comment: an increase of the nucleon axial mass modifies the strength of the response in the quasielastic peak but does not extend the region of response beyond this peak, which is an effect of the multinucleon excitations [93]. Therefore, it could not produce the same spreading effect toward smaller reconstructed neutrino energies as the multinucleon contribution (right panels in Fig. 2). We can conclude that the multinucleon contribution has to be added.

The first three lines in Table II give the $\chi^{2}$, number of degrees of freedom, and goodness of fit of the fit of MiniBooNE data without and with multinucleon interactions in the case of no oscillations. One can see that the goodness of fit is slightly better when multinucleon interactions are taken into account, but it remains very low. In the next section, we consider neutrino oscillations which give a better fit.

\section{TWO-NEUTRINO MIXING}

First, we fitted the MiniBooNE data without and with the multinucleon interactions in the simplest framework of two-neutrino mixing with the oscillation probability

$$
P_{\substack{(-) \\ \nu_{\mu} \rightarrow \nu_{e}}}=\sin ^{2} 2 \vartheta \sin ^{2}\left(\frac{\Delta m^{2} L}{4 E_{\nu}}\right)
$$

where $\Delta m^{2}$ is the squared-mass difference, $\vartheta$ is the mixing angle, $L \simeq 500 \mathrm{~m}$ is the MiniBooNE sourcedetector distance, and $E_{\nu}$ is the neutrino energy. The results of the fit of MiniBooNE data without and with the multinucleon interactions are presented in Table II. One can see that the multinucleon interactions lead to a decrease of the $\chi^{2}$, as expected from the better fit of the low-energy excess. However, the improvement of the fit is rather small, and the best-fit values of $\sin ^{2} 2 \vartheta$ and $\Delta m^{2}$ obtained from the fits of MiniBooNE data without and with the multinucleon interactions are similar. In any case, there is a significant improvement of the fit with oscillations with respect to that without oscillations.

Figure 4 shows a direct comparison of the theoretical excess over the background of $\nu_{e}$ and $\bar{\nu}_{e}$ events in MiniBooNE obtained with neutrino oscillations for some selected values of the oscillation parameters $\sin ^{2} 2 \vartheta$ and $\Delta m^{2}$ without and with the multinucleon interactions. One can see that in all the cases that we have considered the inclusion of multinucleon interactions has the effect of increasing the excess in the low-energy bins, with small decreases in the high-energy bins. Hence, we expect that the inclusion of the multinucleon interactions leads to a better fit of the anomalous low-energy excess. 

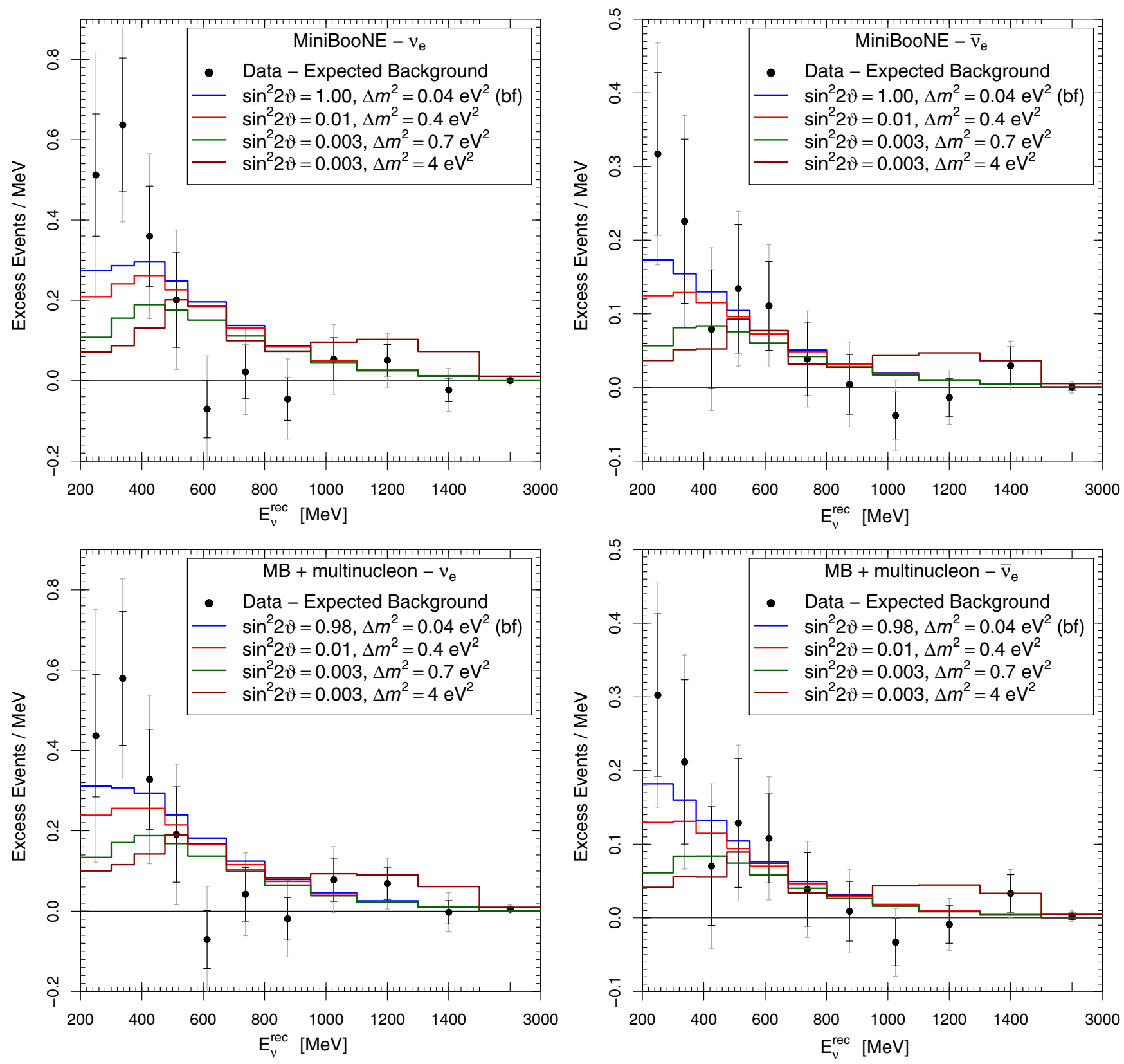

FIG. 5. MiniBooNE excess above the background with statistical (black) and statistical + systematic (gray) error bars in the 11 energy bins of the MiniBooNE data release in neutrino (left) and antineutrino (right) mode without (top) and with (bottom) the multinucleon interactions. The histograms show the predicted excess for the best-fit values and for some other selected values of the oscillation parameters $\sin ^{2} 2 \vartheta$ and $\Delta m^{2}$ indicated by the crosses with corresponding color in Fig. 6.

Figure 5 shows the excess above the background of the MiniBooNE data in the 11 energy bins of the MiniBooNE data release in the neutrino and antineutrino modes without and with the multinucleon interactions. One can see that the low-energy excess slightly decreases when one takes into account the multinucleon interactions, with a small increase of the excess in the intermediate and high-energy bins. This effect is due to the shift shown in Fig. 3 of background events toward lower energies due to the multinucleon interactions. As already discussed for Fig. 3, the change of the excess due to the multinucleon interactions is larger in the neutrino mode than in the antineutrino mode.

The histograms in Fig. 5 show the predicted excess for the best-fit values and for some other selected values of the oscillation parameters $\sin ^{2} 2 \vartheta$ and $\Delta m^{2}$ which lie inside the allowed regions in the $\sin ^{2} 2 \vartheta-\Delta m^{2}$ plane shown in Fig. 6. One can see that for values of the oscillation parameters inside the $1 \sigma$ banana-shaped allowed 

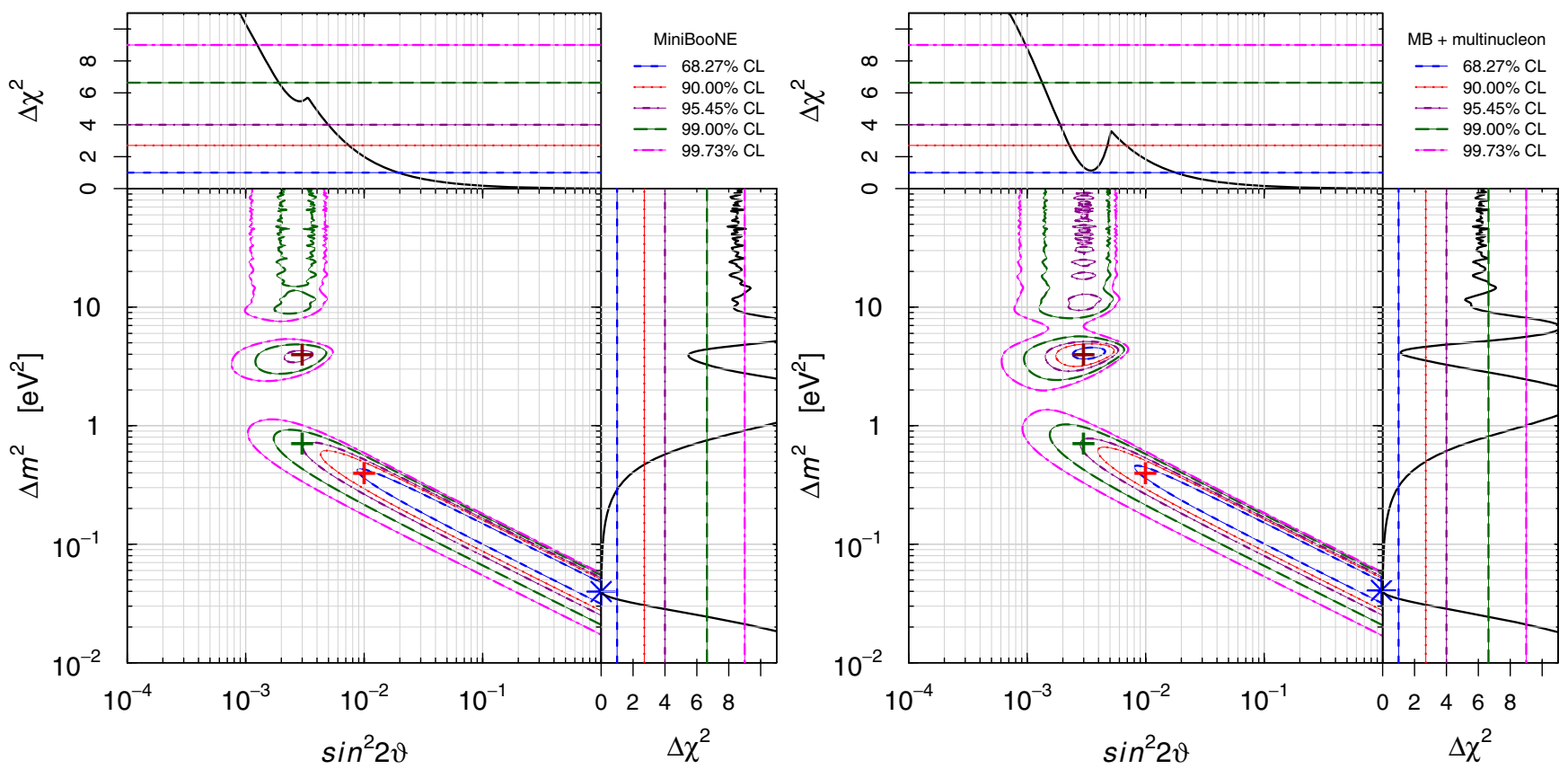

FIG. 6. Allowed regions in the $\sin ^{2} 2 \vartheta-\Delta m^{2}$ plane obtained in a two-neutrino mixing fit of MiniBooNE data without (MiniBooNE) and with $(\mathrm{MB}+$ multinucleon) the multinucleon interactions. The best-fit point is indicated by a blue asterisk. The other crosses indicate the values of $\sin ^{2} 2 \vartheta$ and $\Delta m^{2}$ considered in Fig. 5.

region that goes from $\left(\sin ^{2} 2 \vartheta \simeq 1, \Delta m^{2} \simeq 0.04\right)$ to $\left(\sin ^{2} 2 \vartheta \simeq 0.01, \Delta m^{2} \simeq 0.4\right)$ there is a marginal fit of the low-energy excess in the neutrino mode and this fit is improved when the multinucleon interactions are taken into account. ${ }^{5}$ In the antineutrino mode, there is little change, and the fit of the low-energy excess is marginally acceptable in any case.

However, values of $\sin ^{2} 2 \vartheta$ larger than about 0.003 are excluded by the measurements of several short-baseline $\stackrel{(-)}{\nu}_{e}$ and $\stackrel{(-)}{\nu}_{\mu}$ disappearance experiments, ${ }^{6}$ which did not observe the large $\stackrel{(-)}{\nu}_{e}$ and $\stackrel{(-)}{\nu}_{\mu}$ disappearance which must be

\footnotetext{
${ }^{5}$ The energy distribution of electron-neutrino events in the target depends on the combined variation of the incident muon flux, the oscillation probability and the electron neutrino cross section in the target. All these quantities are rapidly varying with energy. For instance, for $\Delta m^{2} \simeq 0.4$, the transition probability is maximal for $E_{\nu} \simeq 200 \mathrm{MeV}$, where $\Delta m^{2} L / 2 E_{\nu} \simeq \pi / 2$, while the maximum of the energy distribution of the electron events for the same value of $\Delta m^{2}$ occurs at $E_{\nu} \simeq 400 \mathrm{MeV}$. On the other hand, for $\Delta m^{2} \simeq 0.04$, the transition probability is maximal for $E_{\nu} \simeq 20 \mathrm{MeV}$, while the peak of the histogram corresponding to $\left(\sin ^{2} 2 \vartheta \simeq 1, \Delta m^{2} \simeq 0.04\right)$ is also at $E_{\nu}^{\text {rec }} \simeq 400 \mathrm{MeV}$ in the neutrino mode without multinucleon interactions. In this case, the excess of electron-neutrino events is also large because in Eq. (2) the smallness of $\Delta m^{2} L / 2 E_{\nu}$ is compensated by the large value of $\sin ^{2} 2 \vartheta$.

${ }^{6}$ See the red exclusion curve in Fig. 7, which has been obtained in the framework of $3+1$ neutrino mixing.
}

associated with the large $\stackrel{(-)}{\nu}_{\mu} \rightarrow \stackrel{(-)}{\nu}_{e}$ transitions given by $\sin ^{2} 2 \vartheta \gtrsim 0.003$ (see Refs. [2,13-18]). In fact, in the simple two-neutrino framework considered so far, we have

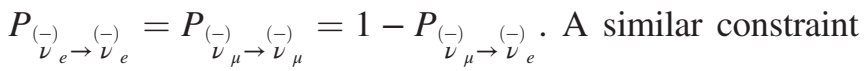
holds in any $3+N_{s}$ neutrino mixing scheme with three active and $N_{s}$ sterile neutrinos [103].

The histograms in Fig. 5 corresponding to $\left(\sin ^{2} 2 \vartheta=0.003, \Delta m^{2}=0.7\right) \quad$ and $\quad\left(\sin ^{2} 2 \vartheta=0.003\right.$, $\left.\Delta m^{2}=4\right)$ show that when the disappearance constraint is taken into account the fit of the MiniBooNE low-energy anomaly is rather bad. There is a small improvement induced by the consideration of the multinucleon interactions, but it is not sufficient to produce an acceptable fit, because the small value of $\sin ^{2} 2 \vartheta=0.003$ gives a prob-

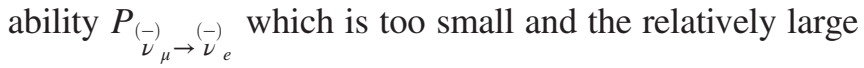
values of $\Delta m^{2}$ lead to a maximum of the event rate which is in the third or a higher energy bin, whereas the maximum of the low-energy excess is in the first two energy bins.

Let us now discuss the implications of our calculations for the allowed regions in the $\sin ^{2} 2 \vartheta-\Delta m^{2}$ plane shown in Fig. 6, where we compare the results of the fits of MiniBooNE data without and with the multinucleon interactions. One can see that the multinucleon interactions induce a significant shift of the allowed regions toward smaller values of $\sin ^{2} 2 \vartheta$ and larger values of $\Delta m^{2}$. In 
particular, the marginal $2 \sigma$ lower bound for $\sin ^{2} 2 \vartheta$ changes from 0.0050 to 0.0019 .

This result indicates that the inclusion of the multinucleon interactions in the analysis of MiniBooNE data may alleviate the appearance-disappearance tension found in the global analyses of the data of short-baseline neutrino oscillation experiments $[16,18,103-112]$. In fact, most of this tension is due to the MiniBooNE low-energy excess, the fit of which requires a small value of $\Delta m^{2}$ and a large value of $\sin ^{2} 2 \vartheta[106,107]$. Decreasing the MiniBooNE low-energy excess by taking into account the multinucleon interactions may lead to a significant improvement of the appearance-disappearance tension.

Let us finally comment on the difference between our results and those presented by the MiniBooNE Collaboration in Ref. [46], which we mentioned in the Introduction. Our method for taking into account multinucleon interactions is an approximate implementation of the correction that one should do to the Monte Carlo event generator, which would result in a correction of the reconstructed neutrino energy $E_{\nu}^{\text {rec }}$ of some events, without any change of the true neutrino energy $E_{\nu}$ of the events, which is determined by the neutrino flux. On the other hand, according to Ref. [113], the MiniBooNE Collaboration simulated the effect of the multinucleon interactions "by reassigning the true neutrino energy of some fraction of events at a given reconstructed neutrino energy to a higher neutrino energy." With their procedure, they obtained a worse fit of the low-energy excess, as shown in Fig. 36 of Ref. [113], and a worse best-fit $\chi^{2}$, given in Table II of Ref. [46], in contradiction with our results.

\section{GLOBAL FIT OF SHORT-BASELINE NEUTRINO OSCILLATION DATA}

To explore if the inclusion of the multinucleon interactions in the analysis of MiniBooNE data decreases the appearance-disappearance tension in the global analyses of the data of short-baseline neutrino oscillation experiments $[16,18,103-112]$, we consider the simplest $3+1$ extension of standard three-neutrino mixing in which there is an additional massive neutrino $\nu_{4}$ with mass $m_{4} \sim 1 \mathrm{eV}$. In this framework, the effective probability of $\stackrel{(-)}{\nu}_{\alpha} \rightarrow \stackrel{(-)}{\nu}_{\beta}$ transitions in short-baseline experiments has the twoneutrino-like form [114]

$$
\underset{\substack{(-) \\ \nu_{\alpha} \rightarrow \nu_{\beta}}}{P_{(-)}}=\delta_{\alpha \beta}-4\left|U_{\alpha 4}\right|^{2}\left(\delta_{\alpha \beta}-\left|U_{\beta 4}\right|^{2}\right) \sin ^{2}\left(\frac{\Delta m_{41}^{2} L}{4 E_{\nu}}\right),
$$

where $U$ is the mixing matrix and $\Delta m_{41}^{2}=m_{4}^{2}-m_{1}^{2}=$ $\Delta m_{\mathrm{SBL}}^{2} \sim 1 \mathrm{eV}^{2}$. The electron and muon neutrino and antineutrino appearance and disappearance in shortbaseline experiments depend on $\left|U_{e 4}\right|^{2}$ and $\left|U_{\mu 4}\right|^{2}$, which
TABLE III. Results of the $3+1$ global fit of short-baseline neutrino oscillation data, taking into account the MiniBooNE data without (MiniBooNE) and with $(\mathrm{MB}+$ multinucleon) the multinucleon interactions. The first three lines give the minimum $\chi^{2}$ $\left[\left(\chi_{\min }^{2}\right)_{\mathrm{GLO}}\right]$, the number of degrees of freedom $\left(\mathrm{NDF}_{\mathrm{GLO}}\right)$, and the goodness of fit $\left(\mathrm{GoF}_{\mathrm{GLO}}\right)$ of the global fit (GLO). The following three lines give the best-fit values of $\Delta m_{41}^{2},\left|U_{e 4}\right|^{2}$, and $\left|U_{\mu 4}\right|^{2}$. The last five lines give the quantities relevant for the appearancedisappearance (APP-DIS) parameter goodness of fit (PG) [115].

\begin{tabular}{lcc}
\hline \hline & MiniBooNE & MB + multinucleon \\
\hline$\left(\chi_{\min }^{2}\right)_{\mathrm{GLO}}$ & 304.0 & 300.7 \\
$\mathrm{NDF}_{\mathrm{GLO}}$ & 268 & 268 \\
$\mathrm{GoF}_{\mathrm{GLO}}$ & $6 \%$ & $8 \%$ \\
$\Delta m_{41}^{2}\left[\mathrm{eV}^{2}\right]$ & 1.6 & 1.6 \\
$\left|U_{e 4}\right|^{2}$ & 0.028 & 0.028 \\
$\left|U_{\mu 4}\right|^{2}$ & 0.014 & 0.015 \\
$\left(\chi_{\min }^{2}\right)_{\mathrm{APP}}$ & 95.4 & 94.0 \\
$\left(\chi_{\min }^{2}\right)_{\mathrm{DIS}}$ & 194.4 & 194.4 \\
$\Delta \chi_{\mathrm{PG}}^{2}$ & 15.0 & 12.7 \\
$\mathrm{NDF}_{\mathrm{PG}}$ & 2 & 2 \\
$\mathrm{GoF}_{\mathrm{PG}}$ & $0.06 \%$ & $0.2 \%$ \\
\hline \hline
\end{tabular}

determine the amplitude $\sin ^{2} 2 \vartheta_{e \mu}=4\left|U_{e 4}\right|^{2}\left|U_{\mu 4}\right|^{2}$ of $\stackrel{(-)}{\nu}_{\mu} \rightarrow \stackrel{(-)}{\nu}_{e}$ transitions, the amplitude $\sin ^{2} 2 \vartheta_{e e}=$ $4\left|U_{e 4}\right|^{2}\left(1-\left|U_{e 4}\right|^{2}\right)$ of $\stackrel{(-)}{\nu}_{e}$ disappearance, and the amplitude $\sin ^{2} 2 \vartheta_{\mu \mu}=4\left|U_{\mu 4}\right|^{2}\left(1-\left|U_{\mu 4}\right|^{2}\right)$ of $\stackrel{(-)}{\nu}_{\mu}$ disappearance.

Table III shows the comparison of the results of the $3+1$ global fit of short-baseline neutrino oscillation data without $^{7}$ and with the multinucleon interactions in the analysis of MiniBooNE data. One can see that in the fit with multinucleon interactions there is a significant improvement of the appearance-disappearance parameter goodness of fit, which quantifies the appearance-disappearance tension. However, this improvement is not sufficient to solve the problem of the appearance-disappearance tension because the value of the appearance-disappearance parameter goodness of fit is still too small.

Figure 7 shows the comparison of the allowed regions in the $\sin ^{2} 2 \vartheta_{e \mu}-\Delta m_{41}^{2}$ plane without and with the multinucleon interactions in the analysis of MiniBooNE data. One can see that the region allowed by appearance data (inside the blue curve) is slightly shifted toward larger values of $\Delta m_{41}^{2}$ by taking into account the multinucleon interactions in the analysis of MiniBooNE data. However, the appearancedisappearance tension persists, since most of the region

\footnotetext{
${ }^{7}$ The results without multinucleon interactions in the "MiniBooNE" column in Table III are different from those in Ref. [18] because we improved the treatment of the MiniBooNE background disappearance due to neutrino oscillations according to information kindly given to us by Bill Louis.
} 

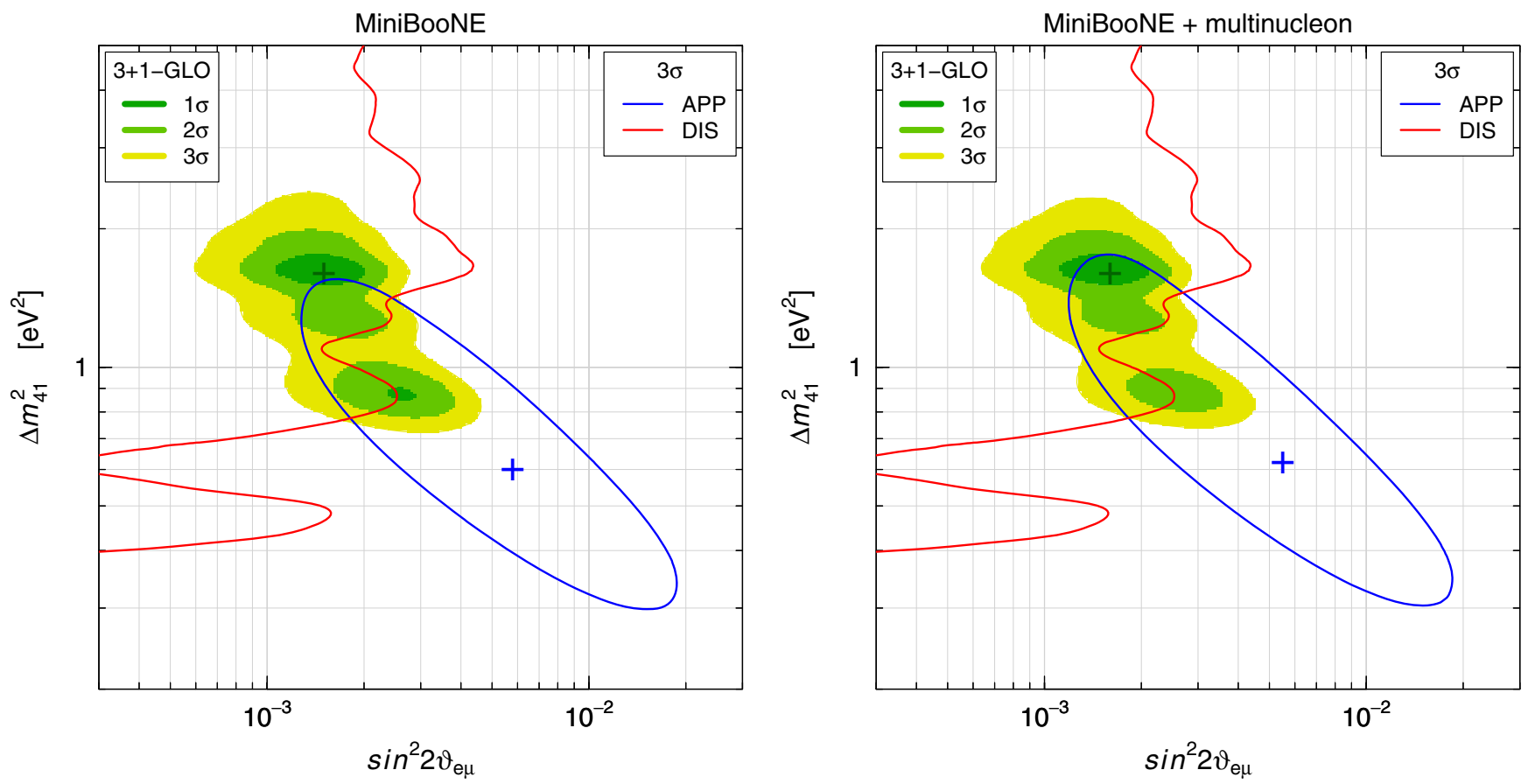

FIG. 7. Allowed regions in the $\sin ^{2} 2 \vartheta_{e \mu}-\Delta m_{41}^{2}$ plane obtained in the $3+1$ global fit of short-baseline neutrino oscillation data, taking into account the MiniBooNE data without (left) and with (right) the multinucleon interactions. In both figures, the region inside the blue curve is allowed at $3 \sigma$ by $\stackrel{(-)}{\nu}_{\mu} \rightarrow \stackrel{(-)}{\nu}_{e}$ appearance data, with the best fit indicated by the blue cross; the region on the right of the red curve is excluded at $3 \sigma$ by $\stackrel{(-)}{\nu}_{e}$ and $\stackrel{(-)}{\nu}_{\mu}$ disappearance data; and the green-yellow shadowed regions are allowed at $1,2,3 \sigma$ by the global fit of all short-baseline neutrino oscillation data.

allowed by appearance data is excluded by the disappearance data (the region on the right of the red curve).

\section{CONCLUSIONS}

In this paper, we have shown that taking into account the multinucleon interactions in the analysis of MiniBooNE data allows a slightly better fit of the MiniBooNE lowenergy excess and induces a shift of the allowed region in the $\sin ^{2} 2 \vartheta-\Delta m^{2}$ plane toward smaller values of $\sin ^{2} 2 \vartheta$ and larger values of $\Delta m^{2}$ in the framework of two-neutrino oscillations.

We performed also a global fit of short-baseline neutrino oscillation data in the framework of $3+1$ neutrino mixing. We have shown that taking into account the multinucleon interactions in the analysis of MiniBooNE data leads to a decrease of the appearance-disappearance tension. However, this effect is not enough in order to solve the problem of the appearance-disappearance tension in the global fit of short-baseline neutrino oscillation data because the value of the appearance-disappearance parameter goodness of fit is still too small.

We conclude that further investigations are needed for solving the puzzle of the MiniBooNE low-energy anomaly. Most notably, the MicroBooNE experiment at Fermilab [116] will check if the MiniBooNE low-energy anomaly is due to photons produced by neutral-current $\nu_{\mu}$ interactions (for example, $\pi^{0}$ production with the detection of only one of the two decay photons). $\nu_{e}$-induced events cannot be distinguished from photon-induced events in the MiniBooNE mineral-oil Cherenkov detector, but they can be distinguished in the MicroBooNE Liquid Argon Time Projection Chamber. Eventually, the SBN experiment at Fermilab [117] will check in a conclusive way the LSND anomaly and the neutrino oscillation explanation of the MiniBooNE data.

\section{ACKNOWLEDGMENTS}

We would like to thank Teppei Katori and Bill Louis for information on the MiniBooNE experiment and M. B. Barbaro for useful discussions. M. V. G. would like to thank the Department of Physics of the University of Torino and the INFN Torino Section for hospitality and partial support. The work of M. V. G. was supported by Deutsche Forschungsgemeinschaft in Sonderforschungsbereich 676. The work of C.G. was partially supported by the research grant Theoretical Astroparticle Physics, Grant No. 2012CPPYP7 under the program PRIN 2012 funded by the Ministero dell'Istruzione, Università e della Ricerca. M. M. acknowledges the support and the framework of the "Espace de Structure et de réactions Nucléaire Théorique" (ESNT, http://esnt.cea.fr) at CEA. 
[1] C. Giunti and C. W. Kim, Fundamentals of Neutrino Physics and Astrophysics (Oxford University Press, Oxford, England, 2007), p. 1.

[2] M. C. Gonzalez-Garcia and M. Maltoni, Phys. Rep. 460, 1 (2008).

[3] G. Bellini, L. Ludhova, G. Ranucci, and F. Villante, Adv. High Energy Phys. 2014, 191960 (2014).

[4] G. Mention, M. Fechner, T. Lasserre, T. A. Mueller, D. Lhuillier, M. Cribier, and A. Letourneau, Phys. Rev. D 83, 073006 (2011).

[5] T. A. Mueller et al., Phys. Rev. C 83, 054615 (2011).

[6] P. Huber, Phys. Rev. C 84, 024617 (2011).

[7] J. N. Abdurashitov et al. (SAGE Collaboration), Phys. Rev. C 73, 045805 (2006).

[8] M. Laveder, Nucl. Phys. B, Proc. Suppl. 168, 344 (2007).

[9] C. Giunti and M. Laveder, Mod. Phys. Lett. A 22, 2499 (2007).

[10] C. Giunti and M. Laveder, Phys. Rev. C 83, 065504 (2011).

[11] C. Giunti, M. Laveder, Y. Li, Q. Liu, and H. Long, Phys. Rev. D 86, 113014 (2012).

[12] A. Aguilar et al. (LSND Collaboration), Phys. Rev. D 64, 112007 (2001).

[13] S. M. Bilenky, C. Giunti, and W. Grimus, Prog. Part. Nucl. Phys. 43, 1 (1999).

[14] A. Strumia and F. Vissani, arXiv:hep-ph/0606054.

[15] K. N. Abazajian et al., arXiv:1204.5379.

[16] J. Conrad, C. Ignarra, G. Karagiorgi, M. Shaevitz, and J. Spitz, Adv. High Energy Phys. 2013, 163897 (2013).

[17] A. Palazzo, Mod. Phys. Lett. A 28, 1330004 (2013).

[18] S. Gariazzo, C. Giunti, M. Laveder, Y. Li, and E. Zavanin, J. Phys. G 43, 033001 (2016).

[19] F. Kaether, W. Hampel, G. Heusser, J. Kiko, and T. Kirsten, Phys. Lett. B 685, 47 (2010).

[20] J. N. Abdurashitov et al. (SAGE Collaboration), Phys. Rev. C 80, 015807 (2009).

[21] C. Athanassopoulos et al. (LSND Collaboration), Phys. Rev. Lett. 75, 2650 (1995).

[22] S. Schael et al. (ALEPH, DELPHI, L3, OPAL, SLD, LEP Electroweak Working Group, SLD Electroweak Group, and SLD Heavy Flavour Group Collaborations), Phys. Rep. 427, 257 (2006).

[23] B. Pontecorvo, Sov. Phys. JETP 26, 984 (1968).

[24] P. C. de Holanda and A. Y. Smirnov, Phys. Rev. D 69, 113002 (2004).

[25] P. C. de Holanda and A. Y. Smirnov, Phys. Rev. D 83, 113011 (2011).

[26] C. Das, J. Pulido, and M. Picariello, Phys. Rev. D 79, 073010 (2009).

[27] S. K. Kang, Y.-D. Kim, Y.-J. Ko, and K. Siyeon, Adv. High Energy Phys. 2013, 138109 (2013).

[28] P. Bakhti and Y. Farzan, J. High Energy Phys. 10 (2013) 200.

[29] A. Palazzo, J. High Energy Phys. 10 (2013) 172.

[30] A. Esmaili, E. Kemp, O. L. G. Peres, and Z. Tabrizi, Phys. Rev. D 88, 073012 (2013).

[31] I. Girardi and D. Meloni, Phys. Rev. D 90, 073011 (2014).

[32] I. Girardi, D. Meloni, T. Ohlsson, H. Zhang, and S. Zhou, J. High Energy Phys. 08 (2014) 057.
[33] A. D. Iura, I. Girardi, and D. Meloni, J. Phys. G 42, 065003 (2015).

[34] F. P. An et al. (DAYA-BAY Collaboration), Phys. Rev. Lett. 113, 141802 (2014).

[35] T. Asaka, S. Blanchet, and M. Shaposhnikov, Phys. Lett. B 631, 151 (2005).

[36] M. Drewes, Int. J. Mod. Phys. E 22, 1330019 (2013).

[37] A. Boyarsky, D. Iakubovskyi, and O. Ruchayskiy, Phys. Dark Univ. 1, 136 (2012).

[38] A. Kusenko, S. Pascoli, and D. Semikoz, J. High Energy Phys. 11 (2005) 028.

[39] G. Gelmini, E. Osoba, S. Palomares-Ruiz, and S. Pascoli, J. Cosmol. Astropart. Phys. 10 (2008) 029).

[40] M. Masip, P. Masjuan, and D. Meloni, J. High Energy Phys. 01 (2013) 106.

[41] H. Ishida, M. Kusakabe, and H. Okada, Phys. Rev. D 90, 083519 (2014).

[42] S. Antusch and O. Fischer, J. High Energy Phys. 05 (2015) 053.

[43] F. F. Deppisch, P. S. B. Dev, and A. Pilaftsis, New J. Phys. 17, 075019 (2015).

[44] S. Antusch and O. Fischer, J. High Energy Phys. 10 (2014) 94.

[45] A. A. Aguilar-Arevalo et al. (MiniBooNE Collaboration), Phys. Rev. Lett. 102, 101802 (2009).

[46] A. Aguilar-Arevalo et al. (MiniBooNE Collaboration), Phys. Rev. Lett. 110, 161801 (2013).

[47] C. Giunti, arXiv:1512.04758.

[48] M. Martini, M. Ericson, and G. Chanfray, Phys. Rev. D 85, 093012 (2012).

[49] M. Martini, M. Ericson, and G. Chanfray, Phys. Rev. D 87, 013009 (2013).

[50] M. Martini, M. Ericson, G. Chanfray, and J. Marteau, Phys. Rev. C 80, 065501 (2009).

[51] M. Martini, M. Ericson, G. Chanfray, and J. Marteau, Phys. Rev. C 81, 045502 (2010).

[52] A. A. Aguilar-Arevalo et al. (MiniBooNE Collaboration), Phys. Rev. D 81, 092005 (2010).

[53] J. Marteau, J. Delorme, and M. Ericson, Nucl. Instrum. Methods Phys. Res., Sect. A 451, 76 (2000).

[54] A. A. Aguilar-Arevalo et al. (MiniBooNE Collaboration), Phys. Rev. D 88, 032001 (2013).

[55] L. Fields et al. (MINERvA Collaboration), Phys. Rev. Lett. 111, 022501 (2013).

[56] G. A. Fiorentini et al. (MINERvA Collaboration), Phys. Rev. Lett. 111, 022502 (2013).

[57] J. Wolcott et al. (MINERvA Collaboration), Phys. Rev. Lett. 116, 081802 (2016).

[58] K. Abe et al. (T2K Collaboration), arXiv:1602.03652.

[59] R. Acciarri et al. (ArgoNeuT Collaboration), Phys. Rev. D 90, 012008 (2014).

[60] T. Walton et al. (MINERvA Collaboration), Phys. Rev. D 91, 071301 (2015).

[61] K. Abe et al. (T2K Collaboration), Phys. Rev. D 91, 112002 (2015).

[62] P. Rodrigues et al. (MINERvA Collaboration), Phys. Rev. Lett. 116, 071802 (2016).

[63] J. Amaro, M. Barbaro, J. Caballero, T. Donnelly, and C. Williamson, Phys. Lett. B 696, 151 (2011). 
[64] J. Nieves, I. R. Simo, and M. J. V. Vacas, Phys. Rev. C 83, 045501 (2011).

[65] A. Bodek and H. Budd, Eur. Phys. J. C 71, 1726 (2011).

[66] M. Martini, M. Ericson, and G. Chanfray, Phys. Rev. C 84, 055502 (2011).

[67] J. Nieves, I. R. Simo, and M. V. Vacas, Phys. Lett. B 707, 72 (2012).

[68] J. Amaro, M. Barbaro, J. Caballero, and T. Donnelly, Phys. Rev. Lett. 108, 152501 (2012).

[69] O. Lalakulich, K. Gallmeister, and U. Mosel, Phys. Rev. C 86, 014614 (2012).

[70] J. Nieves, I. R. Simo, and M. V. Vacas, Phys. Lett. B 721, 90 (2013).

[71] M. Martini and M. Ericson, Phys. Rev. C 87, 065501 (2013).

[72] R. Gran, J. Nieves, F. Sanchez, and M. J. V. Vacas, Phys. Rev. D 88, 113007 (2013).

[73] M. Martini and M. Ericson, Phys. Rev. C 90, 025501 (2014).

[74] G. Megias et al., Phys. Rev. D 91, 073004 (2015).

[75] M. Ericson and M. Martini, Phys. Rev. C 91, 035501 (2015).

[76] M. V. Ivanov, G. D. Megias, R. Gonzalez-Jimenez, O. Moreno, M. B. Barbaro, J.A. Caballero, and T.W. Donnelly, J. Phys. G 43, 045101 (2016).

[77] M. Martini, N. Jachowicz, M. Ericson, V. Pandey, T. Van Cuyck, and N. Van Dessel, arXiv:1602.00230.

[78] J. T. Sobczyk, Phys. Rev. C 86, 015504 (2012).

[79] T. Katori, AIP Conf. Proc. 1663, 030001 (2015).

[80] J. Schwehr, D. Cherdack, and R. Gran, arXiv:1601.02038.

[81] C. Wilkinson et al., arXiv:1601.05592.

[82] J. Nieves, F. Sanchez, I. R. Simo, and M. J. V. Vacas, Phys. Rev. D 85, 113008 (2012).

[83] O. Lalakulich and U. Mosel, Phys. Rev. C 86, 054606 (2012).

[84] T. Leitner and U. Mosel, Phys. Rev. C 81, 064614 (2010).

[85] U. Mosel, O. Lalakulich, and K. Gallmeister, Phys. Rev. Lett. 112, 151802 (2014).

[86] A. M. Ankowski, O. Benhar, and M. Sakuda, Phys. Rev. D 91, 033005 (2015).

[87] A. M. Ankowski, O. Benhar, P. Coloma, P. Huber, C.-M. Jen, C. Mariani, D. Meloni, and E. Vagnoni, Phys. Rev. D 92, 073014 (2015).

[88] P. Coloma and P. Huber, Phys. Rev. Lett. 111, 221802 (2013).

[89] P. Coloma, P. Huber, C.-M. Jen, and C. Mariani, Phys. Rev. D 89, 073015 (2014).

[90] A. A. Aguilar-Arevalo et al. (MiniBooNE Collaboration), Phys. Rev. D 83, 052007 (2011).

[91] K. Abe (T2K Collaboration), Phys. Rev. D 87, 092003 (2013).
[92] K. Abe et al. (T2K Collaboration), Phys. Rev. Lett. 113, 241803 (2014).

[93] W. M. Alberico, M. Ericson, and A. Molinari, Nucl. Phys. A379, 429 (1982).

[94] W. M. Alberico, M. Ericson, and A. Molinari, Ann. Phys. (N.Y.) 154, 356 (1984).

[95] E. Oset and L. L. Salcedo, Nucl. Phys. A468, 631 (1987).

[96] A. A. Aguilar-Arevalo et al. (MiniBooNE Collaboration), http://www-boone.fnal.gov/for_physicists/data_release/ nue_nuebar_2012.

[97] O. Lalakulich and U. Mosel, Phys. Rev. C 87, 014602 (2013).

[98] E. Hernandez, J. Nieves, and M. J. V. Vacas, Phys. Rev. D 87, 113009 (2013).

[99] R. B. Patterson, FERMILAB-THESIS-2007-19.

[100] A. A. Aguilar-Arevalo et al. (MiniBooNE Collaboration), Phys. Rev. D 79, 072002 (2009).

[101] A. A. Aguilar-Arevalo, FERMILAB-THESIS-2008-01.

[102] G. S. Karagiorgi, FERMILAB-THESIS-2010-39.

[103] C. Giunti and E. M. Zavanin, Mod. Phys. Lett. A 31, 1650003 (2016).

[104] J. Kopp, M. Maltoni, and T. Schwetz, Phys. Rev. Lett. 107, 091801 (2011).

[105] C. Giunti and M. Laveder, Phys. Rev. D 84, 073008 (2011).

[106] C. Giunti and M. Laveder, Phys. Rev. D 84, 093006 (2011).

[107] C. Giunti and M. Laveder, Phys. Lett. B 706, 200 (2011).

[108] M. Archidiacono, N. Fornengo, C. Giunti, and A. Melchiorri, Phys. Rev. D 86, 065028 (2012).

[109] M. Archidiacono, N. Fornengo, C. Giunti, S. Hannestad, and A. Melchiorri, Phys. Rev. D 87, 125034 (2013).

[110] J. Kopp, P. A. N. Machado, M. Maltoni, and T. Schwetz, J. High Energy Phys. 05 (2013) 050.

[111] C. Giunti, M. Laveder, Y. Li, and H. Long, Phys. Rev. D 88, 073008 (2013).

[112] G. H. Collin, C. A. Arguelles, J. M. Conrad, and M. H. Shaevitz, arXiv:1602.00671.

[113] G. T. Garvey, D. A. Harris, H. A. Tanaka, R. Tayloe, and G. P. Zeller, Phys. Rep. 580, 1 (2015).

[114] S. M. Bilenky, C. Giunti, and W. Grimus, Eur. Phys. J. C 1, 247 (1998).

[115] M. Maltoni and T. Schwetz, Phys. Rev. D 68, 033020 (2003).

[116] A. M. Szelc (ArgoNeuT and MicroBooNE Collaborations), AIP Conf. Proc. 1666, 180001 (2015).

[117] R. Acciarri et al. (MicroBooNE, LAr1-ND, and ICARUSWA104 COllaborations), arXiv:1503.01520. 